\title{
Exposure to ultrafine particles and respiratory hospitalisations in five European cities
}

\author{
Evangelia Samoli ${ }^{1}$, Zorana Jovanovic Andersen ${ }^{2}$, Klea Katsouyanni ${ }^{1,3}$, \\ Frauke Hennig ${ }^{4}$, Thomas A.J. Kuhlbusch ${ }^{5}$, Tom Bellander ${ }^{6}$, Giorgio Cattani ${ }^{7}$, \\ Josef Cyrys $^{8,9}$, Francesco Forastiere ${ }^{10}$, Bénédicte Jacquemin ${ }^{11,12}$, \\ Markku Kulmala ${ }^{13}$, Timo Lanki $^{14}$, Steffen Loft ${ }^{2}$, Andreas Massling ${ }^{15}$, \\ Aurelio Tobias $^{16}$ and Massimo Stafoggia ${ }^{10}$ on behalf of the UF\&HEALTH \\ Study group
}

Affiliations: ${ }^{1}$ Dept of Hygiene, Epidemiology and Medical Statistics, Medical school, National and Kapodistrian University of Athens, Athens, Greece. ${ }^{2}$ Dept of Public Health, University of Copenhagen, Copenhagen, Denmark. ${ }^{3}$ Dept of Primary Care and Public Health Sciences and Environmental Research Group, King's College London, London, UK. ${ }^{4}$ Leibniz Research Institute for Environmental Medicine, Dusselfdorf, Germany. ${ }^{5}$ IUTA e.V., Air Quality \& Sustainable Nanotechnology Unit, Duisburg and CENIDE, University Duisburg-Essen, Duisburg, Germany. ${ }^{6}$ Institute of Environmental Medicine, Karolinska Institute, Stockholm, Sweden. ${ }^{7}$ Italian National Institute for Environmental Protection and Research, Rome, Italy. ${ }^{8}$ Helmholtz Zentrum München-German Research Center for Environmental Health, Institute of Epidemiology II, Neuherberg, Germany. ${ }^{9}$ Environmental Science Center, University of Augsburg, Augsburg, Germany. ${ }^{10}$ Dept of Epidemiology Lazio Region, Rome, Italy. ${ }^{11}$ INSERM, Aging and Chronic Diseases, Epidemiological and Public Health Approaches, Villejuif, France. ${ }^{12}$ CREAL-Centre for Research in Environmental Epidemiology, Barcelona, Spain. ${ }^{13}$ Division of Atmospheric Sciences, Dept of Physics, University of Helsinki, Helsinki, Finland. ${ }^{14}$ National Institute for Health and Welfare (THL), Kuopio, Finland. ${ }^{15}$ Dept of Environmental Science, Aarhus University, Roskilde, Denmark. ${ }^{16}$ Institute of Environmental Assessment and Water Research (IDAEA), Spanish Council for Scientific Research (CSIC), Barcelona, Spain.

Correspondence: Evangelia Samoli, Dept of Hygiene, Epidemiology and Medical Statistics, National and Kapodistrian University of Athens Medical School, 75 Mikras Asias Street, 11527 Athens, Greece.

E-mail: esamoliamed.uoa.gr

ABSTRACT Epidemiological evidence on the associations between exposure to ultrafine particles (UFP), with aerodynamic electrical mobility diameters $<100 \mathrm{~nm}$, and health is limited. We gathered data on UFP from five European cities within 2001-2011 to investigate associations between short-term changes in concentrations and respiratory hospitalisations.

We applied city-specific Poisson regression models and combined city-specific estimates to obtain pooled estimates. We evaluated the sensitivity of our findings to co-pollutant adjustment and investigated effect modification patterns by period of the year, age at admission and specific diagnoses.

Our results for the whole time period do not support an association between UFP and respiratory hospitalisations, although we found suggestive associations among those $0-14$ years old. We nevertheless report consistent adverse effect estimates during the warm period of the year, statistically significant after lag 2 when an increase by 10000 particles per $\mathrm{cm}^{3}$ was associated with a $4.27 \%$ (95\% CI $\left.1.68-6.92 \%\right)$ increase in hospitalisations. These effect estimates were robust to particles' mass or gaseous pollutants adjustment.

Considering that our findings during the warm period may reflect better exposure assessment and that the main source of non-soluble UFP in urban areas is traffic, our results call for improved regulation of traffic emissions.

@ERSpublications

Exposure to ultrafine particles may increase respiratory hospitalisations in the warm period irrespective of age group http://ow.ly/bGpo300znS1

This article has supplementary material available from erj.ersjournals.com

Received: Dec 142015 | Accepted after revision: April 262016 | First published online: June 232016

Copyright OERS 2016 


\section{Introduction}

Harmful effects of airborne ambient particles on human health are well established [1]. Research has focused on identification of harmful characteristics of particulate air pollution such as particle size, chemical composition and particulate mass, which can be useful for regulation and preventive efforts. However, some toxicological findings suggest stronger effects of smaller particles, particulate matter with aerodynamic diameter $<2.5 \mu \mathrm{m}(\mathrm{PM} 2.5)$, or even smaller particles with diameter $<100 \mathrm{~nm}$, called ultrafine particles (UFPs) compared with larger, coarse particles with diameter 2.5-10 $\mu \mathrm{m}$ (PM2.5-10), or less than $10 \mu \mathrm{m}$ (PM10). However, epidemiological evidence is limited and inconclusive, mainly due to lack of measurements for UFP and coarse particles. More specifically, the measurement of UFP has been developed rather recently and is relatively expensive due to extensive quality assurance guidelines. Furthermore, no national or international regulations are in place to define an upper limit of UFP in ambient air resulting in lack of routinely monitored UFP data. Therefore, only few dedicated measurement campaigns and pilot projects have been conducted to measure UFP concentrations and assess their relationship with health outcomes. As these measurement campaigns are often limited to one city, the few existing epidemiological studies on effects of short-term exposure to UFPs from these sites may lack adequate statistical power, either in Europe [2-4], USA [5] or China [6], and provide mixed results [7].

To close this gap, we undertook a collaborative effort to gather available data on UFP measurements from cities spanned across Europe in order to investigate associations between short-term changes in UFP concentrations and respiratory hospitalisations in children and adults. Five metropolitan areas contributed data to both metrics: Barcelona, Spain; Copenhagen, Denmark; Helsinki, Finland; Rome, Italy; and Stockholm, Sweden. The multi-city time-series design enlarges statistical power to detect weak associations, as is the case in air pollution epidemiology even with non-routinely measured data, provided they are collected over a relatively long time period.

\section{Data and methods}

Data

For each participating city, data were collected on daily counts of emergency respiratory hospital admissions (International Classification of Disease, ICD-9: 460-519; ICD10: J00-J99) with regards to the population resident of the city and hospitalised within the city. Outpatient visits, elective admissions and visits to the emergency department were not included. Only the primary diagnosis was considered for the identification of the outcome. We also collected data on specific respiratory diagnoses, namely lower respiratory tract infections (LRTI; ICD9: 466, 480-487; ICD10: J09-J18, J20-J22), chronic obstructive pulmonary disease (COPD; ICD9: 490-492, 494, 496; ICD10: J40-J44, J47) and asthma (ICD9: 493; ICD10: J45). All data were extracted and collected according to a common protocol. Daily counts were collected for all ages and separately by age groups $0-14$ years, 15-64 years and $>65$ years old. The data covered at least five consecutive years for each city between 2001 and 2011 and in maximum, a period of 11 years (in Copenhagen).

We collected data on available UFP measured in each city as number concentration per $\mathrm{cm}^{3}$ (see online supplement for details on study areas and measurement campaigns, and supplementary tables S1 and S2). The size range of the particles differed between cities. Barcelona measured 5-1000 nm, Copenhagen 6-700 nm, Helsinki 10-100 nm, Rome 7-3000 nm and Stockholm 7-3000 nm during 2001-2005 and 4-3000 nm during 2008-2010. The differences in detected size range, especially the lower size end, will affect the total number counts. Traffic emissions currently display a mode around $10 \mathrm{~nm}$ which can be quite pronounced. Hence a direct comparison of values should be made with care. The location and height of the monitor is also crucial in the determination of UFP levels, as UFP number is amongst the fastest decaying pollutants with distance from traffic due mainly to dilution and agglomeration [8]. In all participating cities, one urban background monitor was used, except in Rome where the monitor was influenced by traffic. Measurement sites were located in the centre of the respective cities, except for in Helsinki where it was located about $5 \mathrm{~km}$ northeast. While all were placed near traffic avenues the height of the site placement differed from $2 \mathrm{~m}$ in Rome to $24 \mathrm{~m}$ in Stockholm. On average over all cities, 30\% of days had missing data during the whole study period.

We also collected data on PM2.5 (24 h), PM10 $(24 \mathrm{~h})$ and gaseous pollutants (sulfur dioxide $\left(\mathrm{SO}_{2} ; 24 \mathrm{~h}\right)$, nitrogen dioxide $\left(\mathrm{NO}_{2} ; 24 \mathrm{~h}\right)$, carbon monoxide $(\mathrm{CO} ; 8 \mathrm{~h})$ and ozone $\left.\left(\mathrm{O}_{3} ; 8 \mathrm{~h}\right)\right)$ following a standardised protocol [9]. Daily air pollution measurements were provided by the monitoring networks established in each city participating in the project. Daily PM2.5-10 was calculated for each station as the difference between

Support statement: A. Tobias was supported by the project PI15/00515, integrated in the National Plan for I+D+I and cofunded by the ISCIII Directorate General for Evaluation and the European Regional Development Fund (FEDER). Funding information for this article has been deposited with the Open Funder Registry.

Conflict of interest: Disclosures can be found alongside this article at erj.ersjournals.com 
PM10 and PM2.5, provided that both PM fractions were measured at the same station using the same sampling methodology. Urban or suburban background sites and fixed monitors located near traffic (only when they represented the exposure of the population living nearby) with at least $75 \%$ complete information for the study period provided the data for the epidemiological analysis. The monitor-specific concentrations were averaged and missing values from the averaged series were imputed by the average of the values of the remaining stations for that day multiplied by a factor equal to the ratio of the annual mean for the missing station over the corresponding annual mean from the other stations [10]. For days with missing values in all used monitoring stations, the resulting series would also have a missing value on that date.

Time series data on daily temperature $\left({ }^{\circ} \mathrm{C}\right.$, daily mean) were used to control for the potential confounding effects of weather. External information on influenza epidemics was also collected.

\section{Methods}

We used a hierarchical modelling approach. First, we fit regression models in each city separately to allow specific control for seasonal effects, weather and other potential confounders. We used the results of the individual city analysis in the second-stage analysis to provide overall estimates using meta-analysis techniques.

The associations between UFPs and other pollutants and respiratory admissions for each city were investigated using Poisson regression models allowing for overdispersion. We used penalised regression splines as a smoothing function for time, with natural cubic splines as base functions. We chose six effective degrees of freedom per 365 days of available data to control for seasonality. To control for weather, we included two smooth terms of temperature in order to better account for both high and low temperatures at different reference lags. For high temperatures, we calculated the average temperature on the current and previous day (lag 0-1) and fit a natural spline with three degrees of freedom on the lagged variable only for days on which the lag $0-1$ temperature was higher than the median annual temperature for the city as a whole. Similarly, we adjusted for low temperatures by fitting a natural spline with two degrees of freedom for the average temperature on previous 6 days (lag 1-6) only for days on which the lag 1-6 temperature was below the median annual value for the city [9]. We also included indicator variables for the day of the week effect, for holidays and the population decrease during the summer vacation periods typical for each city. We controlled for influenza epidemics using an indicator variable denoting days with particularly high influenza episodes from hospitalisation records and surveillance systems.

The pollutant was entered in the model using single day lags. We investigated separately the effect of several lags ranging from the same day with the admission (lag 0) to 10 days before (lag 10). We did not include distributed lag structures in our models as the missing pattern of the data prohibits the interpretation of combined lags.

To investigate potential confounding effects of the daily levels of pollutants, we applied two pollutant models, i.e. we included in the model for UFP alternatively other fractions of particles (PM10, PM2.5, $\mathrm{PM}$ 2.5-10), or gases, $\mathrm{NO}_{2}, \mathrm{SO}_{2}$ or $\mathrm{O}_{3}$. Both pollutants were entered in the model using the same lag. We also investigated the effect modification patterns of the associations between respiratory hospital admission and UFP by age group using stratified analysis.

We explored seasonal effects of UFP on respiratory morbidity by defining the cool period from October to March and the warm period from April to September. We performed a separate analysis by period, fitting a Poisson regression model with three degrees of freedom per 183 days with available data.

In the second stage of the analysis, we assumed the city-specific effect estimates to be normally distributed around an overall estimate. We estimated a fixed effects pooled estimate by giving each city a weight inversely proportional to the variance of its effect estimate. In the presence of heterogeneity, we applied random-effects pooled estimates, where we assumed that city-specific estimates were a sample of independent observations from a normal distribution with the same mean and with variances equal to the sum of the between-city variance and the squared standard error of the city-specific estimate. The random variance component was estimated using the approach by DerSimonian and LAIRD [11]. We applied the Cochran's Q test and the $\mathrm{I}^{2}$ statistic, as proposed by Higgins and THOMPSON [12], to examine heterogeneity. We comment on the random effects estimates, that take into account heterogeneity if present or coincide with fixed effects estimates in case of its absence.

All models were fit using R v.3.0.3 (R development Core Team (2011), ISBN 3-900051-07-0; www.R-project.org). Results in tables and plots are presented as percentage change associated with an increase equal to 10000 particles per $\mathrm{cm}^{3}$ for UFP and $10 \mu \mathrm{g} \cdot \mathrm{m}^{-3}$ for the rest of the pollutants, which roughly correspond to the between-city mean of the pollutants' interquartile ranges. 
TABLE 1 Characteristics of participating cities and descriptive statistics of pollutants, temperature and daily respiratory hospital admissions

\begin{tabular}{|c|c|c|c|c|c|}
\hline & Barcelona & Copenhagen & Helsinki & Rome & Stockholm \\
\hline Study period & $2005-2010$ & $2001-2011$ & $2001-2010$ & $2001-2010$ & $2001-2005$ and $2008-2010$ \\
\hline Population $\times 1000$ & 1602 & 1263 & 1000 & 2548 & 789 \\
\hline UFP particles per $\mathrm{cm}^{3}$ & $19554 \pm 8044$ & $5105 \pm 2563$ & $7951 \pm 4912$ & $34043 \pm 20164$ & $9128 \pm 4320$ \\
\hline $\mathrm{PM}_{10} \mu \mathrm{g} \cdot \mathrm{m}^{-3}$ & $34.9 \pm 17.3$ & $19.5 \pm 9.3$ & $15.7 \pm 9.6$ & $36.0 \pm 15.1$ & $14.8 \pm 8.6$ \\
\hline $\mathrm{PM}_{2.5} \mu \mathrm{g} \cdot \mathrm{m}^{-3}$ & $22.7 \pm 11.4$ & $13.0 \pm 6.9$ & $9.0 \pm 6.3$ & $19.6 \pm 10.0$ & $7.8 \pm 5.0$ \\
\hline $\mathrm{O}_{3} \mu \mathrm{g} \cdot \mathrm{m}^{-3}$ & $62.7 \pm 27.9$ & $32.8 \pm 11.0$ & $62.4 \pm 20.9$ & $72.6 \pm 37.1$ & $64.9 \pm 20.3$ \\
\hline $\mathrm{CO} \mathrm{mg} \cdot \mathrm{m}^{-3}$ & $0.7 \pm 0.4$ & $0.3 \pm 0.1$ & $0.6 \pm 0.4$ & $1.5 \pm 0.9$ & $0.8 \pm 0.7$ \\
\hline Temperature ${ }^{\circ} \mathrm{C}$ & $14.5 \pm 6.1$ & $9.6 \pm 6.9$ & $5.8 \pm 9.5$ & $15.9 \pm 7.1$ & $7.2 \pm 8.0$ \\
\hline $\begin{array}{l}\text { Respiratory admissions rate per } 1000 \text { people year } \\
\text { Respiratory admissions }\end{array}$ & 67.7 & 156.3 & 71.4 & 47.8 & 123.5 \\
\hline All ages & $54 \pm 18$ & $49 \pm 15$ & $20 \pm 7$ & $33 \pm 11$ & $35 \pm 11$ \\
\hline LRTI & $22 \pm 10$ & $22 \pm 9$ & $11 \pm 5$ & $12 \pm 6$ & $18 \pm 8$ \\
\hline COPD & $12 \pm 5$ & $9 \pm 4$ & $2 \pm 2$ & $7 \pm 4$ & $7 \pm 3$ \\
\hline Asthma & $3 \pm 2$ & $4 \pm 2$ & $2 \pm 1$ & $1 \pm 1$ & $2 \pm 2$ \\
\hline
\end{tabular}

Data are presented as mean \pm SD, unless otherwise stated. PMx: particulate matter with aerodynamic diameter <x $\mu$ m; PM2.5-10: particulate matter with aerodynamic diameter 2.5-10 $\mu \mathrm{m}$; UFP: ultrafine particles; LRTI: lower respiratory tract infections; COPD: chronic obstructive pulmonary disease.

\section{Results}

Table 1 shows descriptive characteristics of the data from each participating city during the study period. Particles' mass concentrations and, in particular, UFP number concentrations varied between cities, presenting a rather distinct geographical distribution with a north-to-south gradient. The highest UFP measurements recorded in Rome reflect the influence of traffic near the monitoring site. All cities contributed data for long periods of time that included a lot of missing values for UFP measurements ranging from $4 \%$ in Helsinki to $47 \%$ in Copenhagen. However, the missing values did not follow any distinct seasonal pattern (supplementary figure S1). Stockholm contributed data for two different time periods, 2001-2005 and 2008-2010, that were accounted for using an indicator variable in the respective models. The highest correlations were observed between UFP and $\mathrm{NO}_{2}$ ranging from 0.38 in Barcelona to 0.69 in Helsinki (supplementary table S3), while correlations between UFP and CO ranged from 0.07 in Stockholm and Barcelona to 0.67 in Rome, and between UFP and PM2.5 from 0.09 in Stockholm to 0.57 in Rome. The mean daily number of respiratory admissions ranged from 20 in Helsinki to 54 in Barcelona (supplementary figure S2). The highest numbers of hospitalisations occurred among those aged $>65$ years. Rates displayed heterogeneity partly due to admissions practices associated with differences in primary care as well as different practices in diagnoses. Rates displayed opposite geographical pattern to air pollution concentrations, as southern cities presented lower rates compared with northern cities.

Figure 1 (and supplementary table S4) presents the pooled percentage change (and 95\% confidence intervals) in respiratory hospitalisations in the five European cities per 10000 increase in UFP number concentration per $\mathrm{cm}^{3}$ and $10 \mu \mathrm{g} \cdot \mathrm{m}^{-3}$ in PM10, PM2.5 and PM2.5-10 for individual lags 0-10. Effects of other pollutants were calculated for the same period that UFP measurements were available to ensure comparability. We verified previously reported associations with PM10, PM2.5 and PM2.5-10 mainly at shorter lags up to 5 days prior to the health event, as for example, an increase in previous day (lag 1) PM10 levels by $10 \mu \mathrm{g} \cdot \mathrm{m}^{-3}$ was associated with $0.67 \%$ (95\% CI $\left.0.13-1.22 \%\right)$ increase in respiratory hospitalisations, while for PM2.5, the increase was $0.68 \%$ (95\% CI $0.01-1.36 \%$ ) and for PM2.5-10 0.45\% (95\% CI -0.46-1.37\%). These estimates were rather consistent with those derived when the whole study period (irrespective of UFP availability) was analysed; e.g. the same increase in lag 1 concentrations was associated with $0.57 \%$ (95\% CI $0.12-1.03 \%$ ), $0.53 \%$ (95\% CI $0.08-0.98 \%)$ and $0.38 \%$ (95\% CI $-0.37-1.14 \%)$ respectively. Our results do not support a clear pattern for an association between UFP number concentration and respiratory hospital admissions, as positive effects were estimated only at longer lags, namely 3, 5 and 6, and did not reach the 


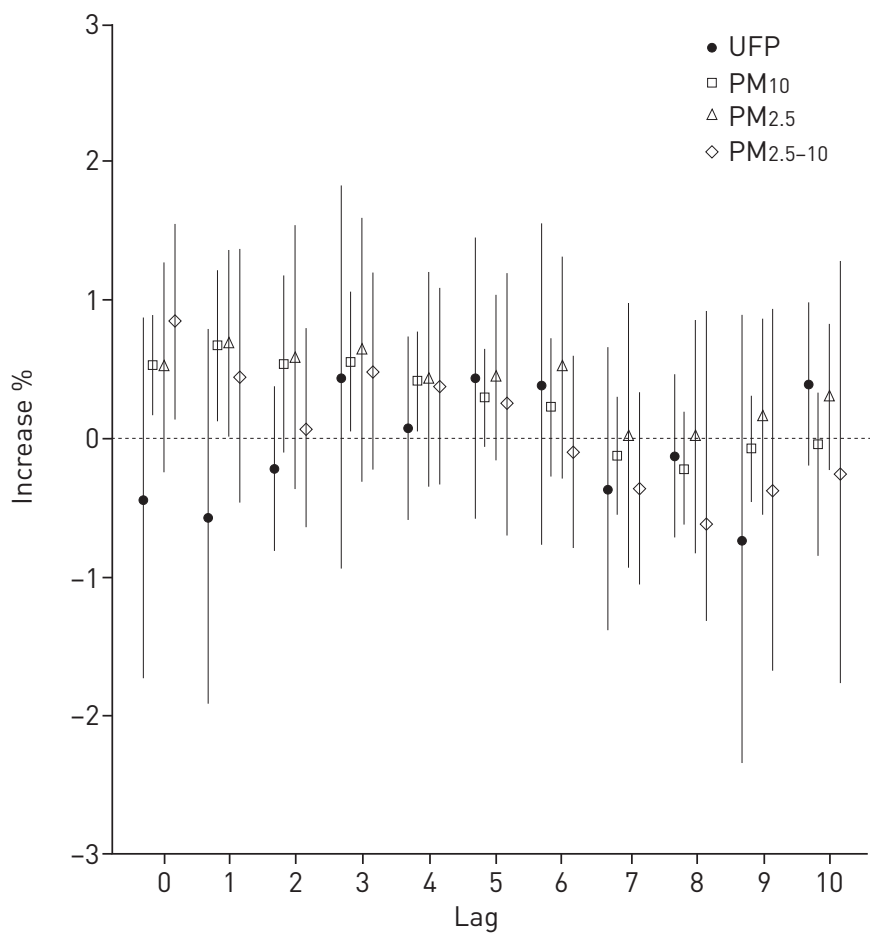

FIGURE 1 Pooled percentage change $(95 \%$ CI) in respiratory hospitalisations in five European cities per 10000 increase in ultrafine particle number concentrations per $\mathrm{cm}^{3}$ and $10 \mu \mathrm{g} \cdot \mathrm{m}^{-3}$ in particulate matter with aerodynamic diameter $<10 \mu \mathrm{m}$ (PM10), <2.5 $\mu \mathrm{m}$ (PM2.5), or 2.5-10 $\mu \mathrm{m}$ (PM2.5-10) for individual lags 0-10.

nominal level of significance. An increase by 10000 in the number of UFP per $\mathrm{cm}^{3}$ was associated with $0.43 \%$ (95\% CI $-0.94-1.83 \%)$ increase in hospitalisations after 3 days. Supplementary figure S3a presents the city specific and pooled estimates for selective lags $2-4$, showing adverse effects in most cities. There was statistically significant heterogeneity in the pooled effects that suggested an association (see supplementary table S5), which could not be explained by geographical region as results remained highly heterogeneous when we pooled estimates separately for the three northern versus the two southern cities.

Table 2 presents the effect estimates for an increase by 10000 particles per $\mathrm{cm}^{3}$ in UFP number concentration on respiratory hospitalisations for single lags 0-7 days, after adjusting for other pollutants at the same lag. UFP estimates, especially in lags 3, 5 and 6 that suggested possible associations from single pollutant models, were partly confounded by PM10, PM2.5 and CO. In these two pollutant models, the magnitude of the estimated effects decreased but nevertheless remained positive in most cases, especially at lags 5 and $6 . \mathrm{NO}_{2}$ completely removed the suggested associations and led to a change in direction, while $\mathrm{NO}_{2}$ effects were robust to UFP adjustment (see supplementary table S6). There did not seem to be confounding by $\mathrm{O}_{3}$.

Table 3 presents the results from the effect modification analysis by age group and selected specific diagnoses for single lags 0-7 days. UFP effect estimates were higher for respiratory admissions among

TABLE 2 Pooled percentage change $(95 \% \mathrm{CI})$ in respiratory hospitalisations in five European cities per 10000 increase in ultrafine particle (UFP) concentration per $\mathrm{cm}^{3}$ adjusting for other pollutants for individual lags 0-7

\begin{tabular}{|c|c|c|c|c|c|c|c|}
\hline Lag & UFP only & +PM10 & +PM2.5 & +PM2.5-10 & $+\mathrm{NO}_{2}$ & $+\mathrm{CO}$ & $+\mathrm{O}_{3}$ \\
\hline 1 & $-0.58(-1.93-0.79)^{\#}$ & $-1.09(-2.50-0.34)$ & $-0.70(-2.39-1.02)$ & $-0.81(-2.46-0.87)$ & $-0.55(-2.16-1.09)$ & $-0.69(-2.12-0.76)$ & $0.08(-1.61-1.80)$ \\
\hline 3 & $0.43(-0.94-1.83)^{\#}$ & $0.13(-1.46-1.76)$ & $0.14(-1.64-1.95)$ & $0.32(-1.35-2.03)$ & $-0.03(-1.43-1.38)$ & $-0.07(-1.52-1.39)$ & $0.69(-0.88-2.29)$ \\
\hline 4 & $0.07(-0.59-0.73)$ & $-0.22(-0.99-0.56)$ & $0.16(-1.14-1.48)^{\#}$ & $0.13(-0.89-1.17)$ & $-0.55(-1.25-0.17)$ & $0.00(-0.75-0.76)$ & $0.63(-0.60-1.87)^{\#}$ \\
\hline 5 & $0.43(-0.58-1.45)^{\#}$ & $0.26(-0.82-1.36)$ & $0.33(-1.17-1.84)$ & $0.43(-0.76-1.64)$ & $-0.82(-1.57--0.07)$ & $0.27(-1.04-1.60)$ & $0.35(-0.57-1.29)$ \\
\hline 6 & $0.38(-0.78-1.56)^{\#}$ & $0.32(-1.02-1.68)$ & $0.14(-1.53-1.84)$ & $0.46(-1.02-1.97)$ & $-0.39(-1.90-1.15)$ & $0.22(-1.31-1.78)$ & $0.52(-0.70-1.75)$ \\
\hline
\end{tabular}

PMx: particulate matter with aerodynamic diameter $<x \mu \mathrm{m}$; PM2.5-10: particulate matter with aerodynamic diameter $2.5-10 \mu \mathrm{m} .{ }^{~}$ : heterogeneity as defined by $\left.\right|^{2}>40 \%$ and $p<0.20$. Bold indicates statistical significance. 
TABLE 3 Pooled percentage change $(95 \% \mathrm{CI})$ in respiratory hospitalisations by age group and for specific diagnoses in five European cities per 10000 increase in ultrafine particle concentration per $\mathrm{cm}^{3}$

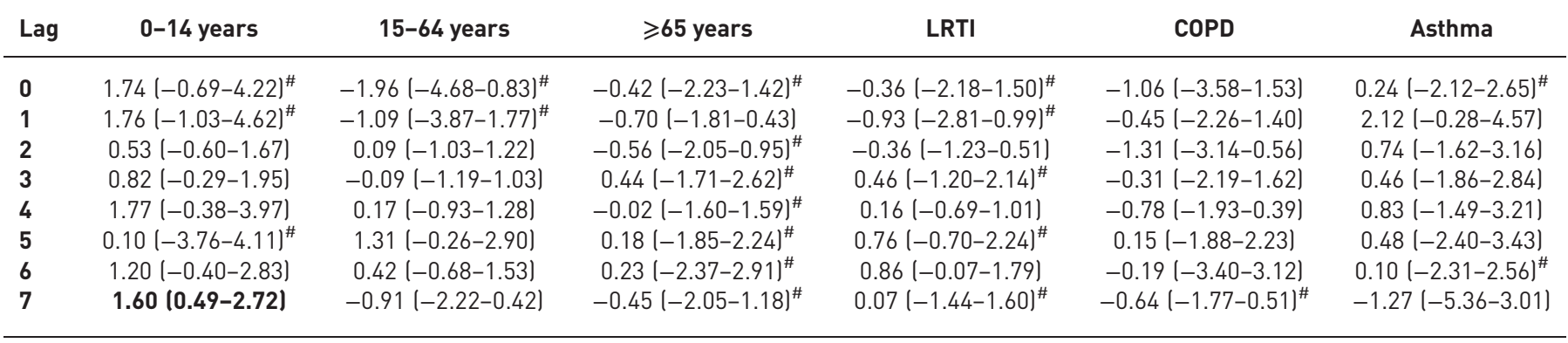

LRTI: lower respiratory tract infections; COPD: chronic obstructive pulmonary disease. ${ }^{\#}$ : heterogeneity as defined by $I^{2}>40 \%$ and $p<0.20$. Bold indicates statistical significance.

children aged 0-14 years irrespectively of the lag under investigation. Regarding specific diagnoses, effect estimates were more consistently positive for asthma in comparison with COPD and LRTI.

Table 4 presents the results from the analysis by cool/warm period of the year for all ages and by age group as it was of interest to see whether the effect modification identified for younger ages presented seasonal patterns. Results indicated consistent effects of UFP on respiratory hospitalisations during the warm period of the year irrespective of age group for all single lags analysed, except lag 0 on 15-64 year olds, while most were also statistically significant. Effect estimates were consistently higher for paediatric hospitalisations $0-14$ years old compared with those aged $>15$ years for both periods. During the cool period of the year, effects were estimated only for paediatric respiratory hospitalisations for almost all lags considered, except lags 1 and 5, which were much lower to the effects detected during the warm period for the corresponding lag. We further investigated the strong effect of UFP during the warm period by allowing for $\mathrm{CO}$ or $\mathrm{NO}_{2}$ in the model. Effect estimates remained positive and significant (in corresponding lags with single pollutant models) after controlling for $\mathrm{CO}$ or $\mathrm{NO}_{2}$, although they decreased in magnitude adjusting for $\mathrm{NO}_{2}$ (see supplementary figure S4). We also tested the sensitivity of the warm period findings to the exclusion of Rome (as the site location differed from the rest) and, although this resulted in some loss of statistical power, the effect estimates were robust especially up to lag 4 . Supplementary figure S3b presents the city specific estimates during the warm period of the year, for selective lags $2-4$, showing adverse effects in all

TABLE 4 Pooled percentage change $(95 \% \mathrm{CI})$ in respiratory hospitalisations for all ages and by age group in five European cities per 10000 increase in ultrafine particle concentration per $\mathrm{cm}^{3}$, stratified by the warm and cool period of the year

\begin{tabular}{|c|c|c|c|c|}
\hline Lags & All ages & $0-14$ years & 15-64 years & $\geqslant 65$ years \\
\hline 0 & $1.80(-0.85-5.42)^{\#}$ & $6.38(1.53-11.46)$ & $-0.19(-4.24-4.04)^{\#}$ & $1.24(-1.40-3.96)^{\#}$ \\
\hline 2 & $4.27(1.68-6.92)^{\#}$ & $6.95(2.21-11.91)$ & $3.11(0.63-5.65)$ & $4.02(0.21-7.98)^{\#}$ \\
\hline 3 & $3.82(2.54-5.12)$ & $5.92(2.74-9.20)$ & $2.82(0.38-5.32)$ & $4.56(1.52-7.68)^{\#}$ \\
\hline 4 & $3.57(1.83-5.34)$ & $6.69(3.51-9.96)$ & $3.15(0.72-5.64)$ & $3.28(0.09-6.57)^{\#}$ \\
\hline 5 & $3.77(1.30-6.30)^{\#}$ & $5.27(-1.68-12.71)$ & $3.83(0.24-7.55)^{\#}$ & $2.85(0.36-5.41)^{\#}$ \\
\hline 7 & $2.36(1.12-3.62)$ & $5.87(-0.60-12.77)$ & $2.07(-1.09-5.34)$ & $1.40(-0.16-2.98)$ \\
\hline \multicolumn{5}{|c|}{ Cool period (October-March) } \\
\hline 0 & $-0.76(-1.87-0.36)$ & $0.59(-1.53-2.76)$ & $-1.82(-4.29-0.71)^{\#}$ & $-1.05(-2.97-0.91)^{\#}$ \\
\hline 1 & $-1.14(-2.37-0.11)$ & $-0.06(-1.33-1.23)$ & $-0.92(-3.32-1.54)^{\#}$ & $-2.13(-4.13--0.09)^{\prime}$ \\
\hline 2 & $-0.90(-1.62--0.17)$ & $0.17(-1.07-1.43)$ & $-0.60(-2.28-1.11)$ & $-1.54(-2.47--0.60)$ \\
\hline 3 & $-0.63(-1.80-0.54)$ & $0.64(-0.59-1.87)$ & $-0.75(-2.00-0.52)$ & $-1.04(-2.67-0.62)$ \\
\hline
\end{tabular}

\#: heterogeneity as defined by $\left.\right|^{2}>40 \%$ and $p<0.20$. Bold indicates statistical significance. 
cities. We additionally tested the robustness of our effect estimates for the whole period, the warm season and the youngest age group, to the exclusion of Helsinki and Copenhagen as accumulation mode particles were not measured in the areas. Our estimates became larger under almost all lags considered with wider associated confidence intervalss (e.g. for lag 5 the estimated increase in all admissions was $0.67 \%$ (95\% CI $-0.86-2.23 \%$ ) for the analysis of the whole period, $4.05 \%$ (95\% CI $0.90-7.30 \%$ ) for the warm period and $1.35 \%$ (95\% CI $-4.40-7.45 \%$ ) for those $0-14$ years old), while in all cases both the direction and the significance of the estimates from the analysis including all cities were not affected.

\section{Discussion}

Using data from five European cities spanning the continent we report indicative associations between UFP and respiratory hospitalisations among the youngest age group of $0-14$ years old and consistent effect estimates during the warm period of the year. Regarding specific diagnoses, we found suggestive associations with asthma admissions that may nevertheless reflect the effects at younger ages, as asthma is a common respiratory disease among this age group.

Most previous studies on the short-term associations between UFP number concentrations and respiratory morbidity have reported weak associations in various lag structures, although each study investigated different respiratory outcomes and age groups, making direct comparison difficult. BRANIš et al. [13] and ATKINSON et al. [2] observed non-statistically significant weak positive associations with respiratory admissions in Prague (Czech Republic) and London (UK) correspondingly, but following different patterns of exposure; the former at lags 0-2 days and the latter at lags 3-6 days. HaLONEN et al. [14] also reported associations between Aitken and accumulation mode particles and respiratory admissions following the average of 5 days exposure in the elderly. PeEL et al. [5] using emergency room visits data in Atlanta (GA, USA) for 1998-2000 for respiratory diseases, did not find an association with UFP number concentrations for all admissions, asthma or COPD for an average exposure over lags 0-2. On the contrary, LeITTE et al. [6] reported increases of emergency visits in Beijing (China), around 2\% for an increase in numbers of 12000 UFP per $\mathrm{cm}^{3}$ in lags $0-2$, while BELLEUDi et al. [15] reported an increase in COPD admissions of $1.59 \%$ (95\% CI $0.03-3.18 \%$ ) per an increase of $~ 9400$ in lag 0 particle number concentrations per $\mathrm{cm}^{3}$ and weaker associations for lags 2 and 3 with LRTI. Finally, HALONEN et al. [4] ISKANDAR et al. [16] and ANDERSEN et al. [17] reported positive associations with paediatric asthma, while the later also reported associations with admissions among the elderly for average exposure over 5 or 6 days. These results support our findings related to asthma admissions but are not in accordance with our results indicating effects mainly for those aged 0-14 and 15-64 years but not for $>65$ years old.

We report considerably higher and statistically significant associations during the warm period of the year for respiratory hospitalisations for all ages and by age group. HALONEN et al. [14] reported stronger associations during May to September among the elderly, while BELLUDI et al. [15] also reported higher effects with LRTI during the spring and fall period. Nevertheless, most of the previous studies did not investigate UFP effects for separate periods of the year. Higher effects of air pollution during the warm period of the year have been previously reported $[9,18]$. The higher associations between UFP number concentrations and respiratory effects in the warm period may be attributed to either changes in exposure conditions, and consequently better exposure characterisation of the population, or changes in the particles' composition. The prior link can be made when considering the time people spend outdoors and enhanced infiltration of outdoor air due to "open windows". The latter is more complicated since the UFP composition changes due to different source contributions as well as different atmospheric chemistry. Sources change by e.g. less house heating contribution and also by photochemistry leading to higher particle number concentrations due to particle formation [19]. Often these particles, formed via gas-phase processes and called secondary particles, are water soluble and less likely to have particles-related toxicity. On the other hand, Jiminez et al. [20] showed the changes in the particle phase in the summer for organic carbon. This carbon is oxidised by photochemistry producing oxidised organic carbon believed to be of higher toxic relevance than the direct emitted organic carbon from natural and anthropogenic sources. Overall, the shift of soot and organic carbon towards traffic, as well as other processes may explain the higher associations observed in the warm period. It is noteworthy that annual effect estimates were mostly driven by the cold period findings that may be partly explained by the greater counts and variability of the outcome during this time of the year.

Our results for the whole period were generally confounded by co-adjustment on other pollutants, mainly those originating from traffic sources, such as $\mathrm{CO}$ and $\mathrm{NO}_{2}$ while the positive effect estimates during the warm period were robust to co-pollutant adjustment. We note that $\mathrm{NO}_{2}$ effects were robust to adjustment of UFP, both for the whole period with available UFP measurements and for the warm period only, supporting the hypotheses of independent effects. Previous results from two pollutant models have reported confounding of UFP effects from $\mathrm{CO}$ and $\mathrm{NO}_{2}$, while the gasses' effects were more robust. 
Nevertheless, these results should be interpreted with caution due to the high correlation of the included pollutants.

We observed significant heterogeneity (supplementary table S5) in many associations under investigation, possibly partly attributable to differences between cities in the lower cut-off size of UPF, as well as the location of the monitoring sites representing an urban background site. Although we could not formally test these hypotheses due to the limited number of cities, when we investigated the sensitivity of our results to the exclusion of Rome, as its site was more influenced by traffic and, in that sense, differed from the rest, heterogeneity was decreased especially in the annual analysis in the lags suggesting associations, while estimates were rather robust (data not shown).

The main strengths of our study include the large time series analysed for each participating city and the multi-city design that provided adequate statistical power over the non-regulated monitoring of UFP, even for the analysis of admissions among those aged 0-14 years for whom counts were smaller compared with the other outcomes. The main limitation of our study is the difference in measurement methods between participating cities, measuring different fraction of particles sizes, as well as the differences in the placement (especially height above ground level) of the background measurement sites at different locations, that probably contributed to the heterogeneity observed in several estimates. Although the number of missing data in some cities was rather high, our findings regarding associations with particles' mass during the period with available UFP measurements were similar to those previously reported $[1,9]$, possibly indicating that the missing patterns have not greatly influenced our pooled estimates. Finally, UFP mainly originate from vehicular exhaust emissions, thus presenting great spatial variability that could not be incorporated under this study design as measurements were drawn from only one background station. Alternatively, CYRYs et al. [21] have noted that the high temporal correlations of particle number concentrations across the city area of Augsburg (Germany) implicated that, in epidemiological time-series studies, the use of one single ambient monitoring site is an adequate approach for characterising exposure to ultrafine particles.

In conclusion, our findings indicate that exposure to UFP particles may increase total hospitalisations due to respiratory diseases during the warm period of the year, especially among those aged 0-14 years. These results add to previous evidence that stricter traffic regulation would result in better protection of public health.

\section{Acknowledgements}

We thank the Consorci Sanitari de Barcelona (Cat-Salut) for providing hospitalisation data for Barcelona and the Agencia Estatal de Meteorologia (Ministerio de Agricultura, Alimentación y Medio Ambiente) for providing the weather data for Spain. We thank the Finnish Meteorological Institute for providing the weather data for Finland and Helsinki Region Environmental Services Authority HSY for providing the air pollution (other than UFP) data for Helsinki, Finland.

The study has been conducted as a collaborative effort of the UF\&HEALTH Study Group. UF\&HEALTH Study Group: S. Breitner, J. Cyrys, R. Hampel, F. Hennig, B. Hoffmann, T. Kuhlbusch; S. Lanzinger, A. Peters, U. Quass, A. Schneider, K. Wolf (Germany); E. Diapouli, K. Elefteriadis, K. Katsouyanni, E. Samoli, S. Vratolis (Greece); T. Ellermann, Z. Ivanovic-Andersen, S. Loft, A. Massling, C. Nordstrøm (Denmark); P.P. Aalto, M. Kulmala, T. Lanki, J. Pekkanen,

P. Tiittanen, T. Yli-Tuomi (Finland); G. Cattani, A. Faustini, F. Forastiere, M. Inglessis, M. Renzi, M. Stafoggia (Italy);

D. Agis, X. Basagaña, B. Jacquemin, N. Perez, J. Sunyer, A. Tobias (Spain); G. Bero-Bedada, T. Bellander (Sweden).

\section{References}

1 WHO. Review of evidence on health aspects of air pollution - REVIHAAP Project. Technical Report. Copenhagen, Denmark, WHO Publications, WHO Regional Office for Europe, 2013.

2 Atkinson RW, Fuller GW, Anderson HR, et al. Urban ambient particle metrics and health: a time-series analysis. Epidemiology 2010; 21: 501-511.

3 Andersen ZJ, Wahlin P, Raaschou-Nielsen O, et al. Size distribution and total number concentration of ultrafine and accumulation mode particles and hospital admissions in children and the elderly in Copenhagen, Denmark. Occup Environ Med 2008; 65: 458-466.

4 Halonen JI, Lanki T, Yli-Tuomi T, et al. Urban air pollution, and asthma and COPD hospital emergency room visits. Thorax 2008; 63: 635-641.

5 Peel JL, Tolbert PE, Klein M, et al. Ambient air pollution and respiratory emergency department visits. Epidemiology 2005; 16: 164-174.

6 Leitte AM, Schlink U, Herbarth O, et al. Size-segregated particle number concentrations and respiratory emergency room visits in Beijing, China. Environ Health Perspect 2011; 119: 508-513.

7 HEI. Review Panel on Ultrafine Particles: Understanding the Health Effects of Ambient Ultrafine Particles. HEI Perspectives 3. Boston, Health Effects Institute, 2013.

8 Karner AA, Eisinger DS, Niemeier DA. Near-roadway air quality: synthesizing the findings from real-world data. Environ Sci Technol 2010; 44: 5334-5344.

9 Stafoggia M, Samoli E, Alessandrini E, et al. Short-term associations between fine and coarse particulate matter and hospitalizations in Southern Europe: results from the MED-PARTICLES project. Environ Health Perspect 2013; 121: 1026-1033.

10 Katsouyanni K, Touloumi G, Samoli E, et al. Confounding and effect modification in the short-term effects of ambient particles on total mortality: results from 29 European cities within the APHEA2 project. Epidemiology 2001; 12: 521-531

11 DerSimonian R, Laird N. Meta-analysis in clinical trials. Controlled Clin Trials 1986; 7: 177-188. 
Higgins JP, Thompson SG. Quantifying heterogeneity in a meta-analysis. Stat Med 2002; 21: 1539-1558.

13 Braniš M, Vyškovská J, Malý M, et al. Association of size-resolved number concentrations of particulate matter with cardiovascular and respiratory hospital admissions and mortality in Prague, Czech Republic. Inhal Toxicol 2010; 22: 21-28.

14 Halonen JI, Lanki T, Yli-Tuomi T, et al. Particulate air pollution and acute cardiorespiratory hospital admissions and mortality among the elderly. Epidemiology 2009; 20: 143-153.

15 Belleudi V, Faustini A, Stafoggia M, et al. Impact of fine and ultrafine particles on emergency hospital admissions for cardiac and respiratory diseases. Epidemiology 2010; 21: 414-423.

16 Iskandar A, Andersen ZJ, Bønnelykke K, et al. Coarse and fine particles but not ultrafine particles in urban air trigger hospital admission for asthma in children. Thorax 2012; 67: 252-257.

17 Andersen ZJ, Loft S, Ketzel M, et al. Ambient air pollution triggers wheezing symptoms in infants. Thorax 2008; 63: $710-716$.

18 Katsouyanni K, Samet JM, Anderson HR, et al. Air pollution and health: a European and North American approach (APHENA). Res Rep Health Eff Inst 2009; 142: 5-90.

19 Kulmala M, Vehkamaäki H, Petäjä T, et al. Formation and growth rates of ultrafine atmospheric particles: a review of observations. Aerosol Sci 2004; 35: 143-176.

20 Jiminez JL, Canagaratna MR, Donahue NM, et al. Evolution of organic aerosols in the atmosphere. Science 2009; 326: 1525-1526.

21 Cyrys J, Pitz M, Heinrich J, et al. Spatial and temporal variation of particle number concentration in Augsburg, Germany. Sci Total Environ 2008; 401: 168-175. 